\title{
TERRESTRIAL GPS POSITIONING SYSTEM
}

\author{
Smriti Sharma ${ }^{1}$, Rajesh Kumar ${ }^{2}$, Pawan Bhadana ${ }^{3}$ \\ ${ }^{1}$ Student, ${ }^{2}$ Lecturer, ${ }^{3}$ HOD, Computer science \& engineering, BSAITM, Haryana, India \\ smritisharma3627@gmail.com, rajeshsingh22@gmail.com,pawanbhadana79@gmail.com
}

\begin{abstract}
Satellite GPS is undoubtedly the most popular and widely used three dimensional positioning technology in the world today, but despite this, cannot provide the positioning requirement in many every day requirements in different environments, such as urban and indoor due to the very weak signals from satellites. Even with high sensitivity GPS receiver cannot be given guaranteed in all situations and accuracies are typically of the order of tens to hundreds of meter at best. Accurate indoor positioning is required for a variety of commercial applications, including warehouse automation, asset tracking, emergency first-responders, and others. In fact, the general expectation of users today is for "GPS-like" positioning performance anywhere they go. Terrestrial GPS positioning is a new positioning technology, developed to address the failure of current satellite technologies for reliable ubiquitous (outdoor and indoor) positioning. In this paper key aspects of the new technology 'terrestrial GPS positioning system' are discussed. Particular emphasis is given on the components like PseudoLite, PseudoNet, Rover (Moving terminal) and TimeLoc and their functionality. The technique and mathematical model used in terrestrial GPS positioning system is described in detail. The Results of installed Terrestrial GPS Positioning Systems are demonstrated and compared with Satellite GPS Positioning System.
\end{abstract}

Index Terms: Indoor GPS system, PseudoLite, PseudoNet, TimeLoc

\section{INTRODUCTION}

The Global Positioning System (GPS) is a reliable, versatile, generally available and comparatively accurate positioning technology, able to operate anywhere across the globe. GPS is, in fact, the most effective general-purpose navigation tool ever developed because of its ability to address a wide variety of applications: air, sea, land, and space navigation; precise timing; geodesy; surveying and mapping; machine guidance/control; military and emergency services operations; hiking and other leisure activities; personal location; and location-based services. These varied applications use different and appropriate receiver instrumentation, operational procedures and data processing techniques. But all require signal availability from a minimum of four GPS satellites for three-dimensional fixes. In the coming decade a number of other Global Navigation Satellite Systems (GNSS), and regional systems and augmentations will be launched. The number of satellites and transmitted signals suitable for centimetre-level accuracy positioning will at least triple. However, the most severe limitation of GPS performance will still remain; the accuracy of positioning deteriorates very rapidly when the user receiver loses direct view of the satellites, which typically occurs indoors or in severely obstructed urban environments. In such environments, the majority of receivers do not function at all, and even the highsensitivity receivers have difficulty in providing coordinates with sub-decameter level accuracies. Accurate indoor positioning is required for a variety of commercial applications, including warehouse automation, asset tracking, emergency first-responders, and others. In fact, the general expectation of users today is for "GPS-like" positioning performance anywhere they go. The inherent limitations of GPS signal availability indoors and in satellite occluded environments, however, has forced researchers to investigate alternative technologies which may be able to replicate GPS/GNSS performance indoors.

Inertial navigation systems (INS) are useful but no panacea because positioning accuracy degrades rapidly with time due to the drift errors of the gyroscopes and accelerometers. Laser or optical-based systems suffer from line-of-sight restrictions, whereas traditional radio navigation-based systems are affected by multipath and time synchronization challenges. The University of New South Wales (UNSW), Sydney (Australia), with a number of academic partners including the University of Nottingham (U.K.), has initiated research in pseudolite in an effort to overcome problems found in GPSoccluded or denied environments. Experiments have included pseudolites in non-synchronous and synchronous modes for a variety of applications, using both the GPS L1 frequency as well as the $2.4 \mathrm{GHz}$ ISM band. A "pseudolite" is a GPS-like signal transmitted by a ground based transmitter or "pseudosatellite". 


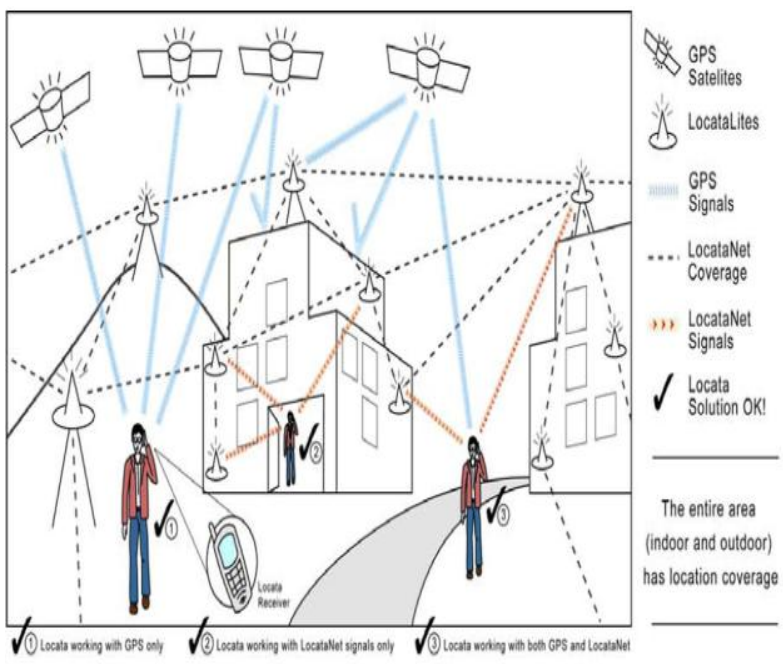

Fig-1: GPS and Terrestrial based Positioning system

\section{TERRESTRIAL GPS POSITIONG}

Terrestrial GPS system is a positioning technology that is designed to overcome the limitations of GPS and other positioning systems currently available. It has invented a timesynchronized pseudolite transceiver called a PseudoLite. A network of Pseudolites forms a PseudoNet, which transmits GPS-like signals that have the potential to allow point positioning with sub-cm precision (using carrier phase) for a mobile unit (Rover). A prototype system has been built to demonstrate the proof-of-concept of the "Terrestrial GPS system technology".

\subsection{PseudoLite}

The PseudoLite can be described as an intelligent pseudolite transceiver. The transmitter prototype hardware used is such that the intelligence of the unit is in software. This is an extremely flexible approach and allows major design changes without requiring completely new hardware. The receiver part of the prototype is based on an existing GPS receiver chipset, and the transmitter share the same clock, which is a cheap temperature compensated crystal oscillator (TCXO). The transmitter part of the prototype generates CA code pseudo range and carrier phase signals at the GPS L1 frequency. The signal is generated digitally and can be operated in a pulsing mode with different duty cycles, power output, and any PRN codes can be generated. Commercially available GPS patch antennas are used for the receiver and transmitter, in addition to a custom built $1 / 4$ wave antenna for one of the PseudoLite transmitters. The prototype PseudoLite and antennas are shown in Figure 2.

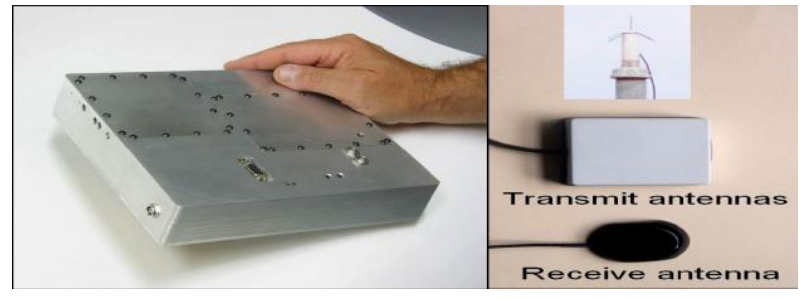

Fig-2: PseudoLite antennas and hardware.

\subsection{TimeLoc}

In order for a mobile receiver to carry out carrier phase point positioning without the need for base station data, the pseudoLite devices must be time-synchronized. The level of synchronization required is extremely high, considering a one nanosecond error in time equates to an error of approximately thirty centimeters (due to the speed of light). The timesynchronization procedure of one or more pseudoLite devices is a key innovation of the "Locata technology" and is known as TimeLoc. The TimeLoc procedure to synchronize one PseudoLite (B) to another PseudoLite (A) can be broken down into the following steps:

1. PseudoLite A transmits a CA code and carrier signal on a particular PRN code.

2. The receiver section of PseudoLite B acquires, tracks and measures the signal (CA code and carrier phase measurements) generated by PseudoLite A.

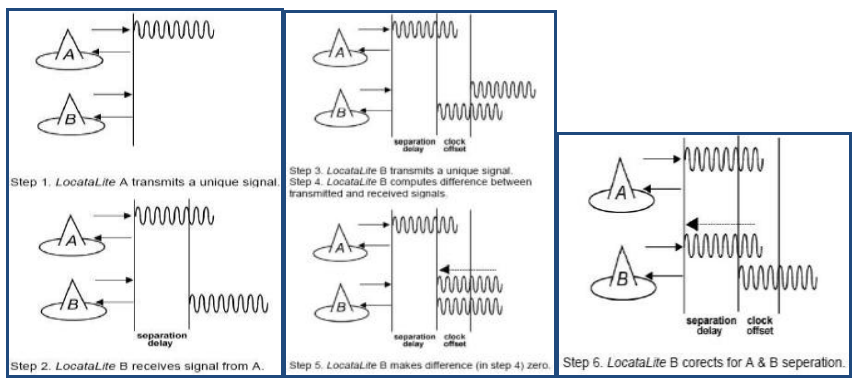

Fig-3: Time synchronization

3. PseudoLite $\mathrm{B}$ generates its own $\mathrm{CA}$ code and carrier signal on a different $\mathrm{PRN}$ code to $\mathrm{A}$.

4. PseudoLite B calculates the difference between the code and carrier of the received signal and its own locally generated signal. Ignoring propagation errors, the differences between the two signals are due to the difference in the clocks between the two devices, and the geometric separation between them.

5. PseudoLite B adjusts its local oscillator using Direct Digital Synthesis (DDS) technology to bring the code and carrier differences between itself and PseudoLite A to zero. The code and carrier differences between PseudoLite A and B are continually monitored so that they remain zero. In other words the local oscillator of B follows precisely that of A. 
The final stage is to correct for the geometrical offset between PseudoLite A and B, and after this TimeLoc is achieved. Importantly, the above procedure does not require expensive atomic clocks and there is no limit to the number of PseudoLites that can be synchronized together using TimeLoc.

In theory, there is no limit to the number of PseudoLites that can be synchronized together using the Time-Loc procedure. This procedure allows a PseudoNet to propagate into difficult environments or over wide areas. For example, if a third PseudoLite C can only receive the signals from B (and not A) then it can use these signals for time-synchronization instead. Moreover, the only requirement for establishing a PseudoNet using Time-LoC is that PseudiLites must receive signals from one other PseudoLites. This does not have to be the same Master LocataLite since this is not possible in difficult environment or when propagating the PseudoNet over wide areas.

\subsection{Mobile Unit (Rover)}

To speed up the development of a prototype system it was decided to use existing GPS hardware for the receiver section in the PseudoLite and the Mobile Unit (the mobile positioning device). Mobile Unit uses the Mitel GP2000 chipset comprised of the GP2015 RF front end and GP2021 12channel correlator together with the P60ARM-B microprocessor. Importantly the system includes GPS firmware $\mathrm{C}$ source code that can be modified, compiled and uploaded to the GPS receiver. However, the GPS Architect hardware is designed as an indoor laboratory development tool and not suited to outdoor use. Instead of designing and building GPS receiver hardware (using the GP2000 chipset), suitable for outdoor use, a different approach was taken. This was to modify a Canadian Marconi Corp (CMC) All star GPS receiver, which uses the Mitel GP2000 chipset, so that it would operate in exactly the same way as the GPS Architect hardware. The original GPS Architect firmware source code has been extensively modified. The modifications have been in signal acquisition, the tracking loops and the navigation algorithm. The prototype hardware and antenna (a commercially available patch antenna) are shown in Figure 2.

\section{MATHEMATICAL MODEL FOR TERRESTRIAL GPS}

The Mobile unit (Rover) uses carrier point positioning (CPP) to determine its three dimensional position from at least four PseudoLites. As the name suggests CPP uses the carrier phase as its basic measurement and it is first useful to consider the carrier phase observations in the case of GPS. The basic GPS L1 carrier phase observation equation between receiver A and satellite $\mathrm{j}$ (in metric units) can be written as:

$$
\begin{aligned}
& \phi_{(A, j)}=\rho_{(A, j}+\tau_{\text {trop }}+c \delta T_{A}-c \delta T_{j}-\tau_{\text {ion }}-\left(c / f_{L I}\right) N_{(A, j)}+\varepsilon \\
& \text { Where, }
\end{aligned}
$$

$f_{L 1} \quad$ is the frequency of the $\mathrm{L} 1$ carrier phase observable;

$c \quad$ is the speed of light in a vacuum;

$\rho_{(A, j)} \quad$ is the geometrical range from station $A$ to satellite $j$;

$\delta T_{A} \quad$ is the receiver clock error for station A;

$\delta T_{j} \quad$ is the satellite clock error for satellite $j$;

$N_{(A, j)} \quad$ is the integer ambiguity (the unknown number of carrier cycles between the receiver $A$ and satellite $j$ at lock-on);

$\tau_{\text {ion }} \quad$ is the atmospheric correction due to the ionosphere;

$\tau \square$ trop $\quad$ is the atmospheric correction due to the troposphere;

$\varepsilon \square \quad$ represents the remaining errors, which may include orbital errors, residual atmospheric effects, multipath error and receiver noise, etc.

For GPS the above equation simply contains too many unknowns to enable a single GPS receiver to perform CPP at the centimetre-level. Instead, terrestrial GPS receiver is used and double-differencing is commonly performed to eliminate both receiver and satellite clock errors, and reduce the effects of the spatially correlated errors due to the troposphere and ionosphere. If real-time GPS positioning using carrier phase is desired the base station data must be available at the rover receiver, usually via a radio modem. The carrier phase integer ambiguities must be determined before centimetre-level carrier-phase positioning can be realized. There are numerous ambiguity resolution approached used, but they can basically be broken down into geometry and geometry-free approaches. However, reliable rapid (less than a minute) On-The-Fly (OTF) ambiguity resolution is only possible when L2 carrier phase data in addition to $\mathrm{L} 1$ is used, and at least five satellites with good geometry are visible.

In comparison to GPS the basic PseudoNet carrier phase observation equation between receiver A and PseudoLite $\mathrm{j}$ (in metric units) can be written as:

$$
\phi_{(\mathrm{A}, \mathrm{j})}=\rho_{(\mathrm{A}, \mathrm{j})}+\tau_{\text {trop }}+\mathrm{c} \delta \mathrm{T}_{\mathrm{A}}-\left(\mathrm{c} / \mathrm{f}_{\mathrm{L} 1}\right) \mathrm{N}_{(\mathrm{A}, \mathrm{j})}+\varepsilon \square
$$

where the terms in the equation are the same as for GPS except they refer to PseudoLites instead of satellites. In the above equation there is no clock error due to the PseudoLites since they are timesynchronised, and because the devices are ground based there is no ionospheric correction term. The tropospheric correction will depend on the separation between the Mobile unit(Rover) and the PseudoLite. The term that poses the most difficulty in the above equation is the unknown number of carrier wavelengths between the Mobile unit(Rover) and the PseudoLite when TimeLoc is achieved. In the prototype system the ambiguity term and the initial receiver clock error are determined through a static initialization at a know point. Assuming that the tropospheric effects are modeled or negligible due to relatively short distances between the Mobile unit(Rover) and PseudoLite, we can write the initial bias (clock error and ambiguity) in metres as:

$$
\begin{gathered}
\mathrm{B}_{(\mathrm{A}, \mathrm{j})}=\mathrm{c} \delta \mathrm{T}_{\mathrm{A}}-\left(\mathrm{c} / \mathrm{f}_{\mathrm{L1}}\right) \mathrm{N}_{(\mathrm{A}, \mathrm{j})}+\varepsilon \\
\mathrm{B}_{(\mathrm{A}, \mathrm{j})}=\rho_{(\mathrm{A}, \mathrm{j})}-\phi_{(\mathrm{A}, \mathrm{j})}
\end{gathered}
$$


The basic observation equation therefore becomes:

and

$$
\phi A j=\rho_{(A, j)}+B_{(A, j)}+c \delta T_{A}+\varepsilon
$$

$$
\rho_{(A, j)}=\operatorname{sqrt}\left(\left(X_{A}-X_{j}\right)^{2}+\left(Y_{A}-Y_{j}\right)^{2}+\left(Z_{A}-Z_{j}\right)^{2}\right)
$$

Where $\square$ TAis the change in the receiver clock error from the static initialization epoch, and this together with the Mobile unit(Rover) coordinates XA ,YA ,ZA give four unknowns; which can be solved with a minimum of four PseudoLite carrier phase measurements and least squares estimation. The least squares estimation procedure is similar to that for standard GPS single point positioning (SPP), except that the very precise carrier phase measurement is used. After the carrier phase bias is determined through static initialisation the Mobile unit(Rover) is free to navigate kinematically. The positioning algorithm is embedded in the GPS firmware of the Pseudo to allow real-time positioning.

\section{EXPERIMENT TO TEST SATELLITE AND TERRESTRIAL POSITIONING}

Dams, bridges, and tall buildings are examples of structures that are routinely surveyed and monitored for their stability. As terrestrial GPS provides centimetre level accurate position solutions with millimetre noise level for static positioning using carrier phase measurements.
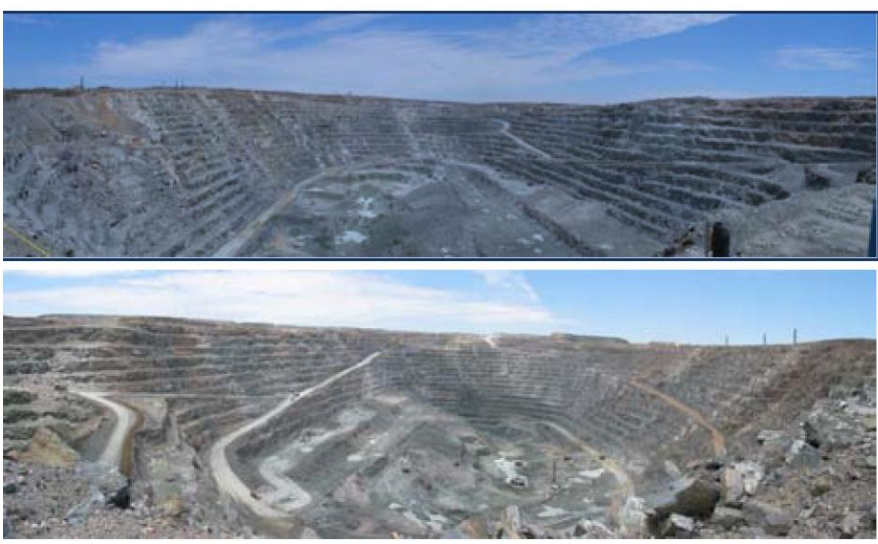

Fig-4: Mine with PseudoLite antenna and PseudoNet

An experiment was done in a diamond mine in South Africa. The Mine pit covers an area of approximately $1.2 \mathrm{X} 0.8 \mathrm{Km}$ and $0.25 \mathrm{Km}$ deep. Photograph of the mine are shown in Fig- 4 . The dimensions of pit at present are such that Satellite GPS positioning availability is approximately $90 \%$ for the majority of pit. This makes the mine an ideal test area to compare Satellite GPS positioning and Terrestrial GPS Positioning. As the pit depth gets deeper the Satellite GPS availability is expected to drop significantly such that mining productivity will be severely affected.
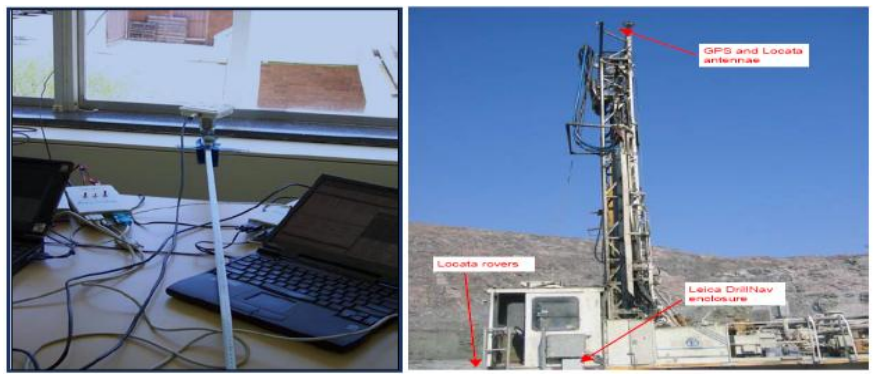

Fig-5: Mobile Terminal (Rover) and PseudoLite.

Mobile Unit (Rover) and PseudoLites can be designed as per site requirement. Mobile Unit will be vehicle mounted and robot mounted which depends upon applications. Similarly, numbers and heights of PseudoLites also vary as per applications. However, in Fig-5 Mobile Unit and PseudoLites installed for experiments are shown.

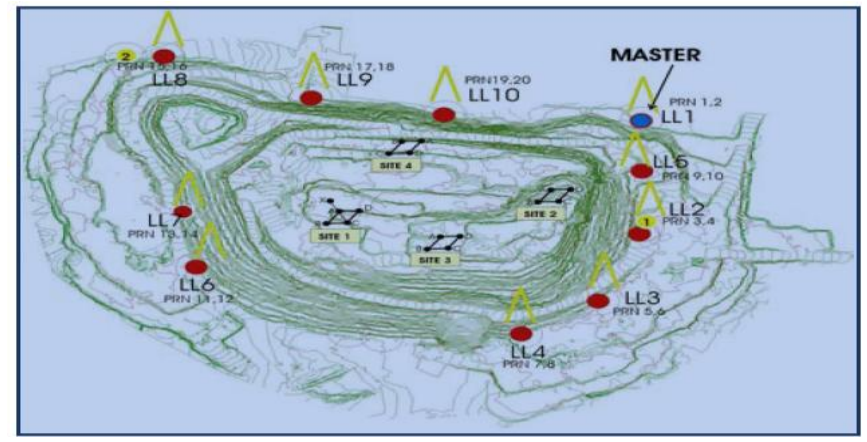

Fig-6: Location of PseudoLites for experiment.

The PseudoNet was established with the help of 10 dual transmit PseudoLites distributed around the rim of pit. Fig-6 shows the location of each PseudoLite (indicated by a red dot) around the rim of the pit, its number (1-10) and the PRNs broadcast by each PseudoLite (1-20). The PseudiLite site locations were selected to provide good coverage into the main pit work area. A drill machine was equipped with Mobile Unit(Rover).

The selection of location of PseudoLites to built PseudoNet is typical work. Survey of each site is required to get correct terrestrial GPS position in all the areas. Master PseudoLites location should be in a position that it could receive good satellite GPS signal for initial synchronization. All PseudoLites should have direct communication with each other to sync the PseudoNet. The Geo navigation System is used to provide navigation guidance information to an operator, in order to follow a predefined drill pattern in mine. This system is integrated with Mobile unit (Rover) with display feature. The lever arm distance between the Satellite GPS and Rover antennae is fixed and well known. Initially, Rover is initialized with using positioning information from the Satellite GPS receiver. After initialization positioning 
information of Rover is entirely independent of Satellite GPS receiver.

During the experiment, data was collected to assess the performance of the Rover in comparison Satellite GPS receiver. For experiments four sites were selected and at each test site a diamond pattern of four points was occupied in succession using rover as shown in Fig-6. At each point on the diamond the rover was stationary for about one minute, and then moved to the next point. These four points were occupied in a number of different trajectories and error statics of Terrestrial GPS against Satellite GPS were computed.

\section{RESULT}

Fig-7 shows typical satellite and terrestrial GPS positioning with East, North and Height components time series. From Fig-7 it is cleared that satellite and Terrestrial positioning time compare to better than $5 \mathrm{~cm}$ in all three positioning components. It should be noted that at some sites there was insufficient satellite coverage to obtain positions but Terrestrial positioning system worked satisfactory.
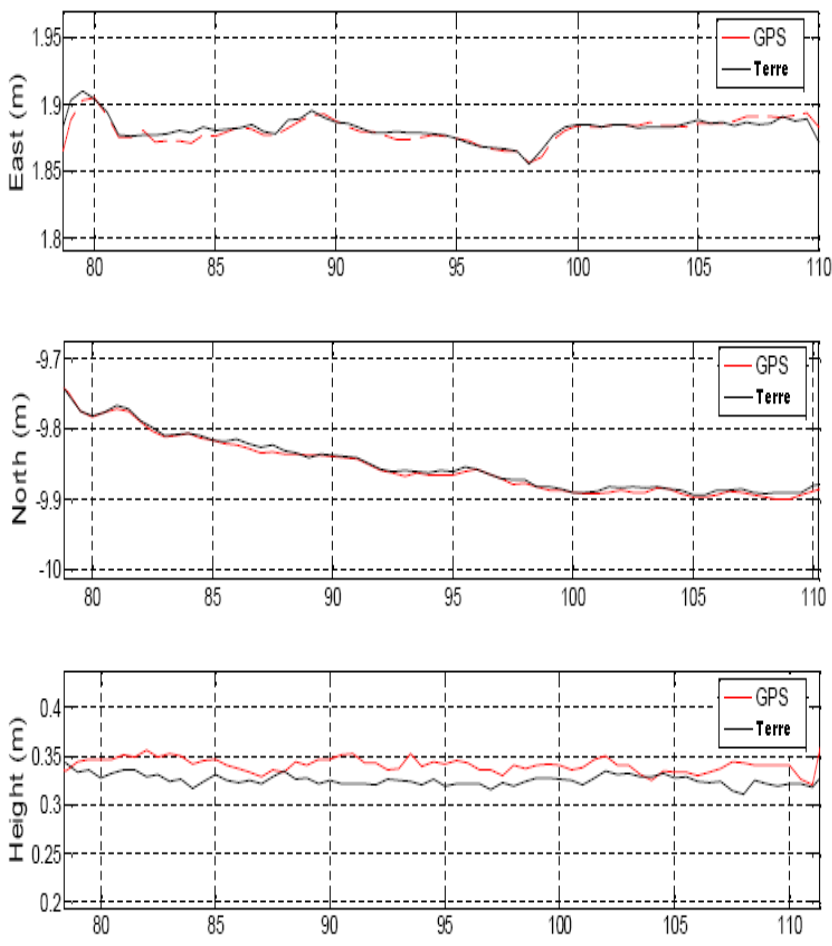

Fig-7: Experiment results.

Interestingly, the satellite GPS statics in the height are better than the horizontal. However, the satellite GPS geometry is also worse at these locations, and suggests that the GPS vertical solution is heavily filtered. The terrestrial GPS is not filtered in either horizontal or vertical components.

\section{CONCLUSIONS}

In this paper the Terrestrial GPS positioning System has been reviewed. It has been shown that the prototype "Terrestrial GPS based technology" clearly demonstrates the proof- ofconcept of a time-synchronised network for positioning. A mathematical model is discussed to validate the usability of this system for tests and field trials. By comparing postprocessed Satellite and Terrestrial GPS positioning it has been demonstrated that accuracy of Terrestrial positioning is comparable to Satellite positioning up to $\mathrm{cm}$-level. Test demonstrates the suitability of Terrestrial GPS for machine guidance, control and tracking applications where there is reduced or unavailable satellite coverage. It is anticipated that this technology will dominate satellite for indoor type activities.

\section{REFERENCES}

[1]. Dai, L, Rizos C, Wang J (2001) The role of pseudosatellite signals in precise GPS-based positioning, Journal of Geospatial Eng., HK Inst. of Engineering Surveyors, 3(1), 33-44.

[2]. Leick A (1995) GPS satellite surveying (second edition), John Wiley \& Sons, Inc., 560pp.

[3]. Yun D, Kee C (2002) Centimeter Accuracy Stand-alone Indoor Navigation System By Synchronized Pseudolite Constellation, 15th International Technical Meeting of the Satellite Division of The Institute of Navigation ION GPS 2002, Portland, Oregon, 24-27 September, 213-225.

[4]. Barnes, J., Wang, J., Rizos, C., \& Tsujii, T., 2002. The performance of a pseudolitebased positioning system for deformation monitoring. 2nd Symposium on Geodesy for Geotechnical \& Structural Applications, Berlin, Germany, 2124 May, 326-337.

[5]. Barnes, J., Cranenbroeck, J. van, Rizos, C., Pahwa, A., \& Politi, A., 2007a. Long term performance analysis of a new ground-transceiver positioning network (LocataNet) for structural deformation monitoring applications. FIG Working Week "Strategic Integration of Surveying Services", Hong Kong, 13-17 May, Session TS5A GNSS2.

[6]. Choudhury, M., Rizos, C., Harvey, B., 2009. A survey of techniques and algorithms in deformation monitoring applications and the use of the Locata technology for such applications. 22nd Int. Tech. Meeting of the Satellite Division of the U.S. Inst. Of Navigation, Savannah, Georgia, USA, 2225 September, 668 - 678.

[7]. Harvey, B.R., 2006. Practical Least Squares and Statistics for Land Surveyors. School of Surveying and Spatial Information Systems, Monograph 13, The University of New South Wales, Sydney, Australia.

[8]. Jäger, R., \& Gonzales, F., 2005. GNSS/GPS/LPS based Online Control and Alarm System (GOCA) - Mathematical Models and Technical Realisation of a System for Natural and Geotechnical Deformation Monitoring and Hazard Prevention. [9]. C. Rizos, D. Grejner-Brzezinska, C.K. Toth, A.G. Dempster, Y. Li, A. Politi, J. Barnes, H. Sun and L. Li. Hybrid 
positioning: A prototype system for navigation in GPSchallenged environments, GPS World, 21(3), 2010, 42-47.

\section{BIOGRAPHIES:}

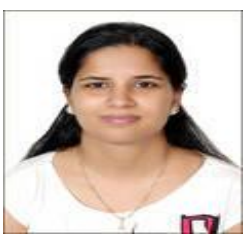

Smriti Sharma is B.Tech., MBA (HRM), $\&$ M.Tech(pursuing). She has 5 yrs of experience in field of education. Six research papers have already been published.

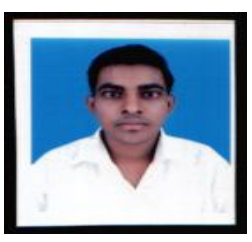

Rajesh Singh is B.Tech ,M.Tech .He has 6 yrs teaching experience .Six research papers have been published till now.

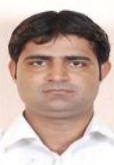

Pawan Bhadana is HOD in BSAITM. He is B.E., M Tech , $\mathrm{PhD}$ (pursuing).he has total $10 \mathrm{yrs}$ of experience in the field of education .total research papers published till now are 15. 